Check for updates

Cite this: Phys. Chem. Chem. Phys., 2020, 22, 12447

Received 30th March 2020 Accepted 6th May 2020

DOI: $10.1039 / \mathrm{d} 0 \mathrm{cp} 01714 \mathrm{k}$

rsc.li/pccp

\section{UV-visible absorption spectrum of FAD and its reduced forms embedded in a cryptochrome protein $\dagger$}

\begin{abstract}
Karno Schwinn, Nicolas Ferré (D) and Miquel Huix-Rotllant (D) *
Cryptochromes are a class of flavoproteins proposed as candidates to explain magnetoreception of animals, plants and bacteria. The main hypothesis is that a biradical is formed upon blue-light absorption by flavin adenine dinucleotide (FAD). In a protein milieu, the oxidized form of FAD can be reduced, leading to four redox derivative forms: anionic and neutral semi-reduced radicals, and anionic and neutral fully reduced forms. All these forms have a characteristic electronic absorption spectrum, with a strong vibrational resolution. Here, we carried out a normal mode analysis at the electrostatic embedding QM/MM level of theory to compute the vibrationally resolved absorption spectra of the five redox forms of FAD embedded in a plant cryptochrome. We show that explicitly accounting for vibrational broadening contributions to electronic transitions is essential to reproduce the experimental spectra. In the case of the neutral radical form of FAD, the absorption spectrum is reproduced only if the presence of a tryptophan radical is considered.
\end{abstract}

\section{Introduction}

Cryptochromes are a class of flavoproteins sensitive to blue light. ${ }^{1}$ A blue sensor is found in their active center, and they usually consist of a flavin adenine dinucleotide (FAD) group. Cryptochromes are found in most living systems, from plants, to animals and bacteria. ${ }^{2,3}$ However, their exact biological role is unknown. They are well known for regulating the circadian clock. ${ }^{4}$ Recently, they have attracted much attention due to their potential role in magnetoreception of certain animals. ${ }^{5,6}$

FAD in protein can exist in five forms: fully oxidized (hereafter simply called $\mathrm{FAD}$ ), an anionic radical form ( $\mathrm{FAD}^{\bullet-}$ ), a neutral radical form $\left(\mathrm{FADH}^{\bullet}\right)$, an anionic reduced form $\left(\mathrm{FADH}^{-}\right)$and a neutral reduced form $\left(\mathrm{FADH}_{2}\right)$. The anionic and neutral forms are connected via acid-base reactions $\left(\mathrm{FAD}^{\bullet}+\mathrm{H}^{+} \leftrightarrow \mathrm{FADH}^{\bullet}\right.$ and FADH $\left.{ }^{-}+\mathrm{H}^{+} \leftrightarrow \mathrm{FADH}_{2}\right)$, while the radical and non-radical forms are connected via a redox reaction $\left(\mathrm{FAD}+\mathrm{e}^{-} \leftrightarrow \mathrm{FAD}^{\bullet-}\right.$ and FADH ${ }^{\bullet}+\mathrm{e}^{-} \leftrightarrow$ FADH $\left.^{-}\right)$. The redox reactions are favored in the excited state (photocatalyst). ${ }^{7}$ The source of protons and electrons to reduce FAD in protein is still uncertain. The most widely accepted hypothesis for plant cryptochromes is that aromatic amino acids in the protein

Aix-Marseille Univ, CNRS, ICR, Marseille, France.

E-mail: miquel.huixrotllant@univ-amu.fr

$\dagger$ Electronic supplementary information (ESI) available: Cartesian coordinates of the optimized QM/MM minimum energy structures for oxidized, anionic and neutral semi-reduced and reduced forms. See DOI: 10.1039/d0cp01714k (probably tryptophan) near the cavity of the cryptochromes transfer electrons and protons to FAD in the excited state. ${ }^{8}$ However, the mechanism might be different in other types of cryptochromes. ${ }^{9}$

The UV-visible absorption spectrum is an essential tool for detecting the different redox states of FAD in protein. Indeed, the five forms have a characteristic absorption spectrum and lifetime. ${ }^{10}$ Simulations of the absorption spectra of FAD and its redox and acid-base derivatives require explicit account of vibrations in order to reproduce the color of absorption and emission. This was first shown by Saalfrank and coworkers for the fully oxidized form of riboflavin. ${ }^{11}$ Recently, several groups have computed the vibrationally resolved absorption spectra for five redox forms of lumichrome- $10 \mathrm{H}$ in the gas phase, ${ }^{12}$ lumiflavin in several solvents, ${ }^{13}$ and the absorption and emission spectra for isoalloxazine, lumichrome, lumiflavin and riboflavin in solution using implicit continuum solvent models. ${ }^{7}$

Even though they are detectable in solution, most of the redox derivatives of FAD (especially for the radical forms) are stabilized in protein. ${ }^{14}$ Currently, a systematic simulation of the absorption spectra of FAD and its redox derivatives in a protein milieu is missing, which would be essential to interpret the origin of the peaks. Cannuccia and coworkers, ${ }^{15}$ and more recently Solov'yov and coworkers ${ }^{16}$ have performed a theoretical study of the vertical transitions at the QM/MM level for the oxidized form of the flavin mononucleotide and $\mathrm{FAD}$, respectively. However, none of these studies included explicitly the effect of nuclear vibrations in their simulations. 
Here, we perform, for the first time, the simulations of the vibrationally resolved electronic absorption spectrum of FAD in its five redox forms in a plant cryptochrome. For this purpose, we have implemented our recently developed method for computing the analytic second derivatives of electrostatic embedding QM/MM energy to unrestricted single-determinant wavefunctions, ${ }^{17}$ necessary to simulate the vibrationally resolved spectra of radical species. Our theoretical absorption spectra are in good agreement with the experimental spectra. For $\mathrm{FADH}^{\bullet}$, the spectra of tyrosil and tryptophanyl radicals have to be included to reproduce the experimental spectra.

\section{Methodology}

Simulations of the vibrationally resolved spectra require the computation of the Hessian for the whole QM/MM system. Currently, there are few methods capable of computing efficiently the QM/MM Hessian for a large macromolecule like a protein, mostly relying on approximations. ${ }^{18-22}$ We have recently developed one of the first analytic electrostatic embedding QM/MM Hessians, which is both efficient and without approximations. $^{23}$ Here, we summarize the main equations following the electrostatic potential fitting (ESPF) method and its derivatives, focusing on the new implementation for unrestricted wavefunctions. The reader is referred to previous literature for further details on the closed shell ESPF equations and analytic $\mathrm{QM} / \mathrm{MM}$ interaction energy derivatives. ${ }^{17,23-25}$

\subsection{Electrostatic potential fitting method}

The ESPF method is an electrostatic embedding QM/MM method, ${ }^{24}$ which adds a one-electron potential in the usual (gas-phase) Fock operator $\left(F_{0}\right)$,

$$
\hat{F}=\hat{F}_{0}+\hat{h} \text {. }
$$

The ESPF Hamiltonian is constructed from a (truncated) multipole order expansion. The lowest-order ESPF Hamiltonian $(\hat{h})$ is given by

$$
\hat{h}=\sum_{\mathrm{A}}^{N_{\mathrm{QM}}}\left(Z_{\mathrm{A}}-\hat{Q}_{\mathrm{A}}\right) \phi_{\mathrm{A}}
$$

where $N_{\mathrm{QM}}$ is the number of $\mathrm{QM}$ atoms, $Z_{\mathrm{A}}$ is the nuclear charge, $\phi_{\mathrm{A}}$ is the external electrostatic potential felt by QM atom $A$ and the atomic charge operator, the matrix elements of which are defined as $Q_{\mathrm{A}}=\sum\left[\left(\mathbf{T}^{\dagger} \mathbf{T}\right)^{-1} \mathbf{T}^{\dagger}\right]_{\mathrm{A}, k} \mathbf{V}_{k}$. Here, $\mathbf{V}_{k}$ are the electrostatic integrals calculated on a grid surrounding the QM subsystem and $\mathbf{T}$ the electrostatic kernel. ${ }^{24}$ Usually, the lowest order approximation is a good approximation for the QM/MM interaction. ${ }^{25}$ Hereafter, we focus on the lowest order, despite being straightforward to generalize it to higher orders in the multipolar expansion. ${ }^{23}$

The ESPF charge operator is spin independent, so the only spin dependency comes from the density matrix. For a single determinant wavefunction expanded in molecular orbitals, the ESPF QM/MM energy is then obtained as

$$
E=\operatorname{Tr}\left[\mathbf{P F}_{0}\right]+E_{\mathrm{MM}}+\operatorname{Tr}[\mathbf{P h}]=E_{0}+E_{\mathrm{MM}}+E_{\mathrm{ESPF}},
$$

in which $\mathbf{P}=\mathbf{P}^{\alpha}+\mathbf{P}^{\beta}$ is the atomic orbital density matrix, and $\mathbf{P}^{\sigma}=\sum_{i}^{N_{\text {occ }, \sigma}} \mathbf{C}_{i}^{\sigma} \mathbf{C}_{i}^{\sigma, \dagger}(\sigma=\alpha, \beta)$. The $\mathbf{C}$ terms are the molecular orbital coefficients obtained from the solution of the secular equation with the Fock operator defined in eqn (1). The ESPF energy interaction term can be rewritten as

$$
E_{\mathrm{ESPF}}=\operatorname{Tr}[\mathbf{P h}]=\sum_{\mathrm{A}}^{N_{\mathrm{QM}}} q_{\mathrm{A}} \phi_{\mathrm{A}}
$$

in which we defined the atomic charge as the sum of the ESPF charge and the nuclear charge,

$$
q_{\mathrm{A}}=Z_{\mathrm{A}}-\operatorname{Tr}\left[\mathbf{P} \mathbf{Q}_{\mathrm{A}}\right] \text {. }
$$

In these equations, the classical analogue of the energy expression eqn (3) becomes apparent.

In the case of unrestricted wavefunctions, the $\alpha$ and $\beta$ contributions to the ESPF charges can be defined as $Q_{\mathrm{A}, \sigma}=$ $\operatorname{Tr}\left[\mathbf{P}^{\mathbf{\sigma}} \mathbf{Q}_{\mathrm{A}}\right]$. Since classical force fields are spin-independent, the ESPF energy expression eqn (4) remains unchanged, and the total atomic charge approximating the QM atom A charge distribution is simply the sum of the $\alpha$ and $\beta$ components,

$$
q_{\mathrm{A}}=Z_{\mathrm{A}}-Q_{\mathrm{A}, \alpha}-Q_{\mathrm{A}, \beta} .
$$

\subsection{Analytic second derivatives of the ESPF energy}

The analytic first and second derivatives of the ESPF energy have been described in ref. 24 and 17, respectively. The ESPF energy depends on the coordinates of the QM and MM atoms. Therefore, its second derivative with respect to these coordinates contains four blocks,

$$
\nabla^{2} E=\left[\begin{array}{ll}
E_{0}^{x y}+E_{\mathrm{MM}}^{x y}+E_{\mathrm{ESPF}}^{x y} & E_{0}^{x \tilde{y}}+E_{\mathrm{MM}}^{x \tilde{y}}+E_{\mathrm{ESPF}}^{x \tilde{y}} \\
E_{0}^{\tilde{x y}}+E_{\mathrm{MM}}^{\tilde{x} y}+E_{\mathrm{ESPF}}^{\tilde{x y}} & E_{0}^{\tilde{x} \tilde{y}}+E_{\mathrm{MM}}^{\tilde{x} \tilde{y}}+E_{\mathrm{ESPF}}^{\tilde{x} \tilde{y}}
\end{array}\right],
$$

in which $E^{x y} \equiv \frac{\partial^{2} E}{\partial x \partial y} . x$ corresponds to the $x$ coordinate of a QM atom, while the tilde symbolizes an MM atom. The analytic second derivatives of $E_{0}$ have been discussed in depth in the literature, ${ }^{26}$ while the $E_{\mathrm{MM}}$ derivatives have a trivial expression. Here we focus on the derivative of the ESPF energy term, which can be written in orders of external potential derivatives as

$$
\begin{aligned}
\nabla^{2} E_{\mathrm{ESPF}}= & {\left[\begin{array}{cc}
\sum_{\mathrm{A}} q_{\mathrm{A}} \phi_{\mathrm{A}}^{x y} & \sum_{\mathrm{A}} q_{\mathrm{A}} \phi_{\mathrm{A}}^{x \tilde{y}} \\
\sum_{\mathrm{A}} q_{\mathrm{A}} \phi_{\mathrm{A}}^{\tilde{x} y} & \sum_{\mathrm{A}} q_{\mathrm{A}} \phi_{\mathrm{A}}^{\tilde{x} \tilde{y}}
\end{array}\right] } \\
& +\left[\begin{array}{cc}
\sum_{\mathrm{A}}\left(q_{\mathrm{A}}^{y} \phi_{\mathrm{A}}^{x}+q_{\mathrm{A}}^{(x)} \phi_{\mathrm{A}}^{y}\right) & \sum_{\mathrm{A}} q_{\mathrm{A}}^{x} \phi_{\mathrm{A}}^{\tilde{y}} \\
\sum_{\mathrm{A}} q_{\mathrm{A}}^{y} \phi_{\mathrm{A}}^{\tilde{x}} & \sum_{\mathrm{A}} q_{\mathrm{A}}^{\tilde{x}} \phi_{\mathrm{A}}^{\tilde{y}}
\end{array}\right] \\
& +\left[\begin{array}{cc}
\sum_{\mathrm{A}} q_{\mathrm{A}}^{(x y)} \phi_{\mathrm{A}} & 0 \\
0 & 0
\end{array}\right],
\end{aligned}
$$


in which the derivatives in parentheses mean the derivatives at the fixed MO coefficients. The first term in eqn (8) corresponds to variations in the MM external field at fixed QM atomic charges (the unique term in mechanical embedding methods), which is equivalent to the second-derivative of the classical electrostatic interaction. The second term and third terms include the charge responses to external field variations, which is due to the fact that the ESPF atomic charges are fluctuating depending on the system MM geometry. In other words, the ESPF charges are polarizable.

The atomic charge derivatives $q_{\mathrm{A}}^{x}$ and $q_{\mathrm{A}}^{\tilde{x}}$ include the derivatives of the density matrix and of the ESPF charge matrix,

$$
\begin{aligned}
& q_{\mathrm{A}}^{x}=-\operatorname{Tr}\left[\mathbf{P}^{x} \mathbf{Q}_{\mathrm{A}}\right]-\operatorname{Tr}\left[\mathbf{P} \mathbf{Q}_{\mathrm{A}}^{x}\right] \\
& q_{\mathrm{A}}^{\tilde{x}}=-\operatorname{Tr}\left[\mathbf{P}^{\tilde{x}} \mathbf{Q}_{\mathrm{A}}\right]-\operatorname{Tr}\left[\mathbf{P} \mathbf{Q}_{\mathrm{A}}^{\tilde{x}}\right]
\end{aligned}
$$

The second equation requires, in principle, the construction of the density matrix derivatives with respect to MM perturbations. The latter are usually obtained by solving a set of coupled-perturbed equations for all MM perturbations. Since the number of MM atoms is usually much larger than the number QM atoms, we have introduced in ref. 23 an auxiliary set of coupled-perturbed equations, the Q-vector equations, which allow computing of this term at an almost negligible cost. The Q-vector equations scale with the number of QM atoms, and take the usual form of the coupled perturbed equations

$$
\sum_{j b}(A-B)_{i a, j b} \tilde{Q}_{\mathrm{A}, j b}=-Q_{\mathrm{A}, i a}
$$

in which $i, j$ are the indexes of the occupied molecular orbitals, and $a, b$ are the indexes of the virtual orbitals, and $(A-B)$ is the usual response matrix (for an explicit expression, see eqn (13) of ref. 23). The solution of this equation, $\tilde{Q}_{\mathrm{A}, j b}$, is then used to construct the ESPF charge derivative with respect to the MM atoms,

$$
q_{\mathrm{A}}^{\tilde{x}}=-2 \sum_{\mathrm{B}, i a} Q_{\mathrm{A}, i a} \phi_{\mathrm{B}}^{\tilde{x}} \tilde{Q}_{\mathrm{B}, i a}
$$

In the case of the $\mathrm{QM} / \mathrm{MM}$ energy second derivatives based on an unrestricted determinant, we can use similar arguments as we did in eqn (6). Since the force field derivatives do not depend on the spin, all charge and charge derivatives of eqn (8) involve the sum of the $\alpha$ and $\beta$ contributions. The Q-vector equation in eqn (10) splits into two expressions

$$
\begin{gathered}
\sum_{j b, \sigma=\alpha, \beta}(A-B)_{i a, \alpha, j b, \sigma} \tilde{Q}_{\mathrm{A}, j b, \sigma}=-Q_{A, i a, \alpha} \\
\sum_{j b, \sigma=\alpha, \beta}(A-B)_{i a, \beta, j b, \sigma} \tilde{Q}_{\mathrm{A}, j b, \sigma}=-Q_{\mathrm{A}, i a, \beta} .
\end{gathered}
$$

in which $\sigma=\alpha, \beta$. Accordingly, the total charge derivative with respect to the MM coordinates becomes

$$
q_{\mathrm{A}}^{\tilde{x}}=-\sum_{\mathrm{B}, i a, \sigma=\alpha, \beta} Q_{\mathrm{A}, i a, \sigma} \phi_{\mathrm{B}}^{\tilde{x}} \tilde{Q}_{\mathrm{B}, i a, \sigma}
$$

\section{Computational details}

The ESPF QM/MM hessian for unrestricted wavefunctions described above has been implemented in a modified version of Gaussian16 interfaced with a modified version of Tinker 6.3.3. ${ }^{17,23,24,27,28}$ The X-ray structure of Arabidopsis Thaliana plant cryptochrome (RCSB PDBID: 2J4D) has been used as a molecular model. Hydrogen atoms have been added to the standard protonation state at physiological $\mathrm{pH}$. For the QM computations, the B3LYP/TZVP/TZVPFit level of theory has been employed for DFT and TD-DFT calculations. ${ }^{29-35}$ For the MM computations, the Amber99 force field has been used, ${ }^{36}$ with van der Waals parameters for FAD taken from ref. 37 . The vibrationally resolved spectrum for FAD has been obtained by Fourier transforming the time-dependent auto-correlation function for the multi-dimensional harmonic oscillator, as implemented in Gaussian16. ${ }^{27,38}$ The vertical gradient approximation has been used in all cases. ${ }^{39}$

\section{Results}

The QM/MM model is defined as FAD (QM subsystem) and the protein scaffold (MM subsystem), from which crystallographic water molecules have been excluded (see Fig. 1). The overall system has been optimized with a quadratically convergent algorithm for the QM part and microiterations for the MM part. From the minimum energy structure of FAD, the subsequent structures have been constructed for the reduced forms and each of them reoptimized. All structures have been confirmed via a frequency calculation, leading to a positive semidefinite internal Hessian matrix in all cases.

\subsection{Geometries}

In the series $\mathrm{FAD}^{\stackrel{\mathrm{e}^{-}}{\rightarrow}} \mathrm{FAD}^{\bullet-} \stackrel{\mathrm{H}^{+}}{\longrightarrow} \mathrm{FADH}^{\bullet} \stackrel{\mathrm{e}^{-}}{\rightarrow} \mathrm{FADH}^{-} \stackrel{\mathrm{H}^{+}}{\longrightarrow}$ $\mathrm{FADH}_{2}$, the lowest occupied molecular orbital (LUMO) becomes a singly occupied molecular orbital (SOMO) after the first electron attachment and the highest occupied molecular orbital (HOMO) after the second electron attachment. The orbital involved in this transformation is essentially antibonding between $\mathrm{N}_{5}-\mathrm{C}_{4 a}$ and bonding between $C_{4 a}-\mathrm{C}_{10 a}$. This transforms the $\alpha$-diimine bond $\left(\mathrm{N}_{5}=\mathrm{C}_{4 a}-\mathrm{C}_{10 a}=\mathrm{N}_{1}\right)$ of the oxidized form in ethene-1,2-diamine $\left(\mathrm{N}_{5}-\mathrm{C}_{4 a}=\mathrm{C}_{10 a}-\mathrm{N}_{1}\right)$ into the fully reduced form. ${ }^{7}$

In Fig. 2, the effect of the protein scaffold on the chromophore optimized (local) minimum energy structure is compared with respect to the gas phase minimum energy structure. For the protein, this corresponds to a local minimum of the internal energy, which is taken as reference to do the harmonic expansion. Overall, one observes only minor structural differences when the chromophore is embedded in the protein compared to the gas phase. This is evident for bond distances, in which changes of less than $0.1 \AA$ are observed for all five forms. Conversely, angles and dihedral angles show important variations for all cases. This is especially important in the case of the fully reduced forms, $\mathrm{FADH}^{-}$and $\mathrm{FADH}_{2}$. These forms 


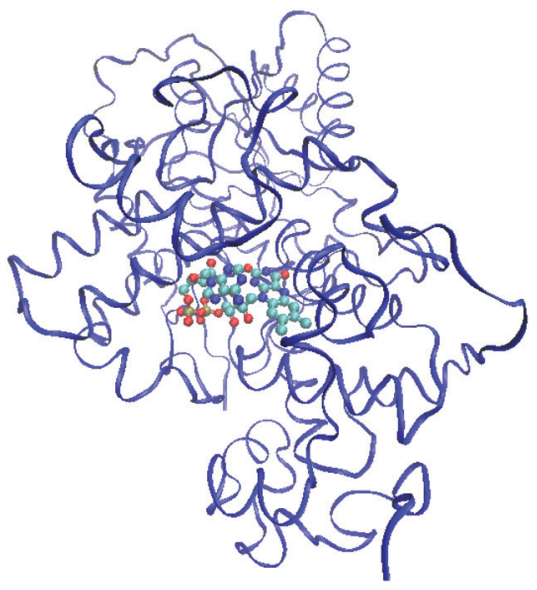

Fig. 1 QM/MM subsystems for Arabidopsis Thaliana cryptochrome (PDBID: 2J4D). The QM fragment representing FAD is shown as balls and sticks, while the MM fragment representing the protein scaffold is shown as ribbons.

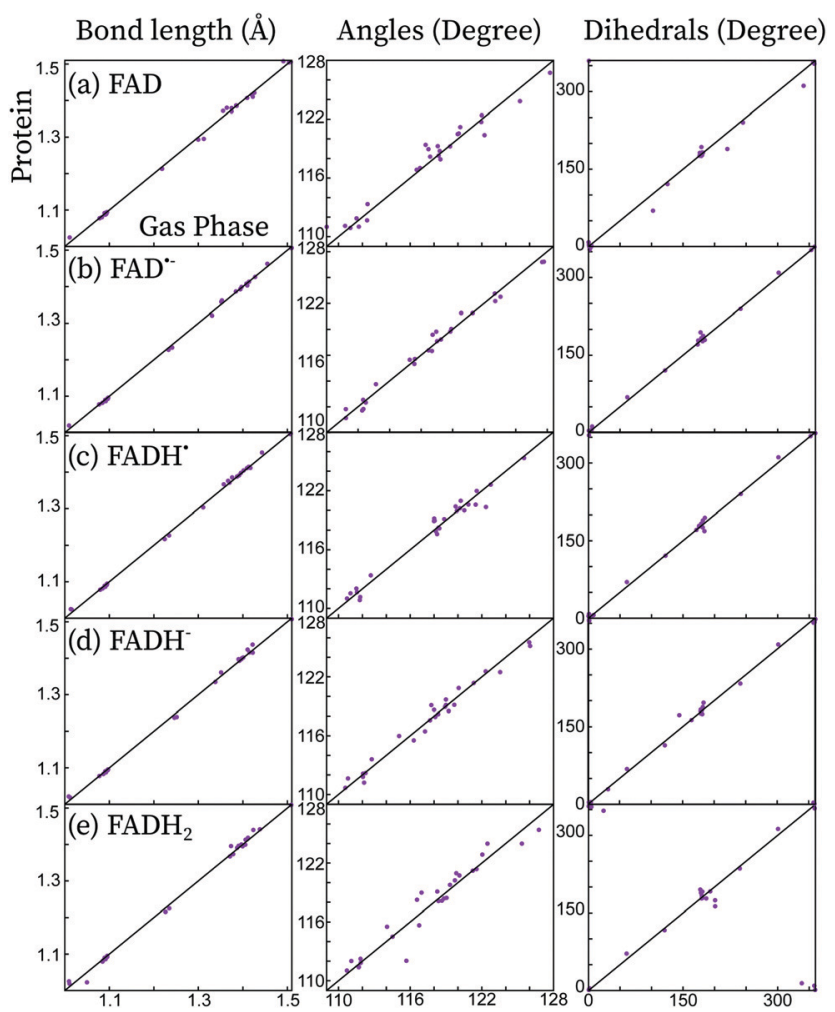

Fig. 2 Comparison of the distances, angles and dihedral angles of the isoalloxazine chromophore optimized in protein and in the gas phase for the five redox forms considered here. The diagonal line $(y=x)$ is shown as guideline.

contain secondary amines at centre $\mathrm{N}_{5}$ and/or $\mathrm{N}_{1}$ atoms. Secondary amines tend to break the planarity of the chromophore both in the gas phase or in solution (see Fig. 3).,13 Indeed, in the gas phase these two forms find their minimum energy structure in the so-called "butterfly" bending conformation. This structural feature is hindered when the chromophore is

\section{Gas phase Protein}

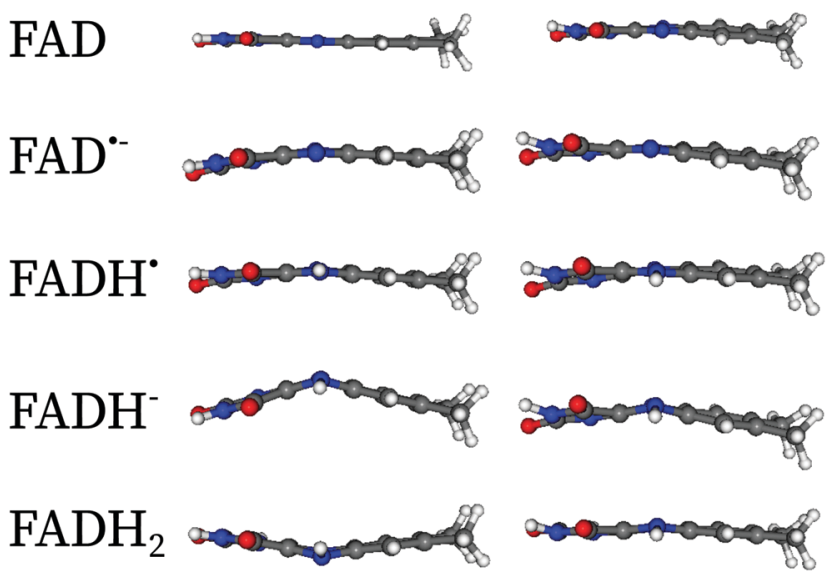

Fig. 3 Comparaison of the minimum energy structures in the gas phase and in protein embedding the isoalloxazine fragment of FAD along the $x z$-plane. The butterfly structures in the gas phase are hindered by the presence of the protein. The Cartesian coordinates of the optimized structures in protein can be found in the ESI. $\dagger$

embedded in the protein, which favours a rather planar structure. Despite the weak electrostatic effects on the $\pi$ system and thus the chromophore excitations, ${ }^{13}$ the conformational constraints have a strong impact on the peak position (see further in the next sections).

\subsection{Absorption spectra of FAD and its redox derivatives embedded in the protein}

In Fig. 4, the total theoretical vibrationally resolved absorption spectrum is shown in the range between $300 \mathrm{~nm}$ and $800 \mathrm{~nm}$. Besides, the contributions of the most important electronic configurations are also explicitly shown. For all redox forms, the lowest-energy peak is represented by a single electronic transition of the type $\pi \rightarrow \pi^{*}$ centred on the isoalloxazine ring. The second peak of $\mathrm{FAD}, \mathrm{FAD}^{\bullet-}$ and $\mathrm{FADH}^{\bullet}$ shows a complex pattern, involving three to four electronic transitions. For $\mathrm{FADH}^{-}$and $\mathrm{FADH}_{2}$ the second transition finds its maximum above $300 \mathrm{~nm}$, and it corresponds to a $\pi \rightarrow \pi^{*}$ transition in both cases.

For all redox forms of FAD, the frontier molecular $\pi_{3}$ orbital plays a central role. This orbital exhibits bonding character between $\mathrm{C}_{4 a}-\mathrm{C}_{10 a}$ and anti-bonding character between $\mathrm{N}_{10}=\mathrm{C}_{10 a}$. For $\mathrm{FAD}, \pi_{3}$ is the lowest unoccupied molecular orbital (LUMO). For the radical forms, $\pi_{3}$ is a singly occupied molecular orbital (SOMO). For the reduced forms, $\pi_{3}$ is the highest occupied molecular orbital (HOMO). For the oxidized and anionic radical forms, a $\pi_{2} \rightarrow \pi_{3}$ transition is observed in the region of $450 \mathrm{~nm}$. This transition is red-shifted in the series $\mathrm{FAD} \rightarrow \mathrm{FAD}^{\bullet-} \rightarrow \mathrm{FADH}^{\bullet}$ since the energetic gap between $\pi_{2}$ and $\pi_{3}$ becomes smaller when FAD is reduced. Indeed, the absorption maxima are predicted at around $460 \mathrm{~nm}, 440 \mathrm{~nm}$ and $650 \mathrm{~nm}$ for $\mathrm{FAD}, \mathrm{FAD}^{\bullet-}$ and $\mathrm{FADH}^{\bullet}$, respectively, corresponding to an absorption color ranging from dark blue, to blue 
(a) FAD

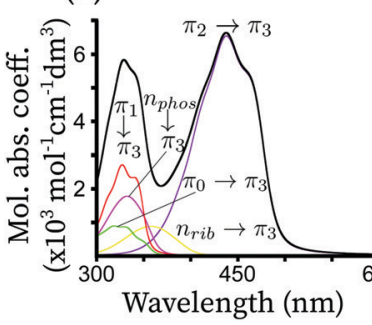

$\begin{array}{ll}\text { (b) } \mathrm{FAD}^{\circ-} & \text { (c) } \mathrm{FADH}^{\circ}\end{array}$

(d) $\mathrm{FADH}^{-}$

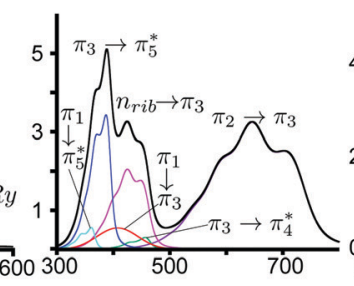

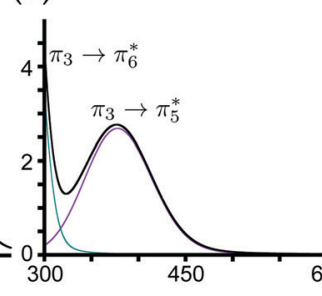

(e) $\mathrm{FADH}_{2}$

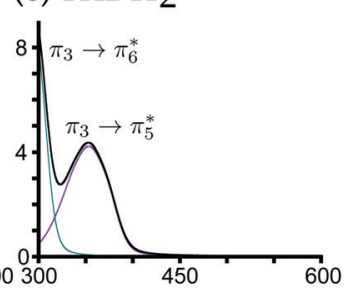

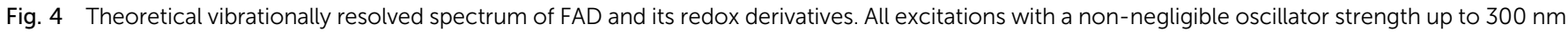

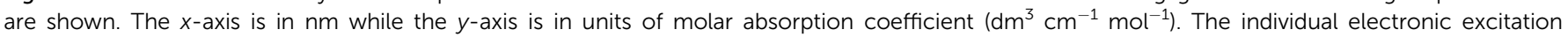

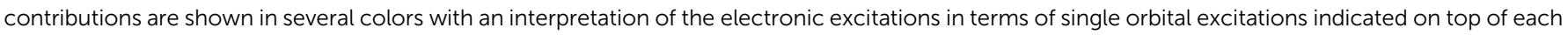
contribution. The total spectrum (black) corresponds to the sum of all individual contributions.

and finally red. For the fully reduced forms, $\pi_{3} \rightarrow \pi_{5}{ }^{*}$ becomes the bright transition, which is blue-shifted with respect to the first transition of the oxidized and semi-reduced forms. Indeed, the absorption maxima are found in the near-UV, around $325 \mathrm{~nm}$ for $\mathrm{FADH}^{-}$and $345 \mathrm{~nm}$ for $\mathrm{FADH}_{2}$. All these transitions have a similar intensity, corresponding to a broad bright peak of $100 \mathrm{~nm}$.

A second intense peak appears between $300 \mathrm{~nm}$ and $800 \mathrm{~nm}$ for the $\mathrm{FAD}, \mathrm{FAD}^{\bullet-}$ and $\mathrm{FADH}^{\bullet}$ species. For the reduced forms, this peak is located outside the considered range, between $200 \mathrm{~nm}$ and $300 \mathrm{~nm}$, corresponding to a $\pi_{3} \rightarrow \pi_{6}{ }^{*}$ transition. For the oxidized and radical forms, this peak is found around $300 \mathrm{~nm}$ and $500 \mathrm{~nm}$ and it includes 3 to 4 different electronic transitions. For the radical forms, the most intense transition corresponds to the electron transfer from the SOMO $\pi_{3}$ to the vacant orbital $\pi_{5}{ }^{*}$. This transition is the same as the first bright transition for the reduced forms, although it is around $50 \mathrm{~nm}$ blue-shifted for the radical species. Indeed, the $\pi_{3} \rightarrow \pi_{5}{ }^{*}$ absorption maximum is found at around $340 \mathrm{~nm}$ for $\mathrm{FAD}^{\bullet-}$. The same transition is found at $360 \mathrm{~nm}$ for $\mathrm{FADH}^{\circ}$. This type of transition is not present in FAD, as in this form, $\pi_{3}$ is a vacant orbital. For FAD, $\pi_{3}$ accepts electrons from the lower lying $\pi$ orbitals and from the oxygen lone-pairs located on the phosphate $\left(\mathrm{n}_{\mathrm{phos}}\right)$ and on the ribitol $\left(\mathrm{n}_{\text {rib }}\right)$. These transitions, which would normally be dark, appear as the peaks of a similar intensity of the $\pi \rightarrow \pi^{*}$ transitions due to two main reasons: (i) these transitions are strongly mixed with the $\pi \rightarrow \pi^{*}$ transitions, thus becoming slightly bright thanks to an intensity-borrowing mechanism, and (ii) the wavefunction overlap between the ground and excited states is large. A similar trend is observed for the radical forms.

\section{Discussion}

The UV-visible absorption spectra of flavins and derivatives (riboflavin, lumichrome, lumiflavin, isoalloxazine, etc.) have been explored in depth in the literature, both experimentally ${ }^{10,40-46}$ and theoretically. ${ }^{11-13,15,16}$ Theoretically, these have been studied mainly in the gas phase and within an implicit description of the solvent. Some of these forms, especially for the radical forms, are usually stabilized when embedded in the protein. To our knowledge, only two theoretical studies have attempted to compute the absorption spectrum in protein, the work of Cannuccia et al..$^{15}$ and the work of Solov'yov et al., ${ }^{16}$ albeit only for the oxidized form and without explicitly accounting for the vibrational structure. Here, we attempt to compare the theoretical absorption spectra in protein and in the gas phase with the experimental spectra of FAD and its redox derivatives in protein. This is shown in Fig. 5. Since many flavoproteins share similar spectral properties, ${ }^{47}$ some of the experimental data have been taken from different forms of cryptochromes or photolyases: data for FAD and $\mathrm{FAD}^{\boldsymbol{\bullet}^{-}}$have been taken from Mosquito Cryptochrome 1 (Anopheles gambiae), and FADH ${ }^{\bullet}$ and $\mathrm{FADH}^{-}$from a photolyase of $E$. Coli. The four forms are similar to the absorption spectrum of Arabidopsis Thaliana cryptochrome 1 and have been taken from ref. 45. The spectrum of $\mathrm{FADH}_{2}$ has been taken from solution measurements, and it is reproduced from the data taken from ref. 10. In the following, we discuss each form separately.

\section{FAD}

The oxidized form of FAD is the most stable one, both in solution and in protein. For this reason, it has been extensively studied in the literature both experimentally ${ }^{41,44,46,48-55}$ and theoretically. ${ }^{7,11,13,16,56-64}$ FAD has two main absorption bands, the first band in the blue visible light region and the second band in the UV-A region. In protein, the first is centered around $450 \mathrm{~nm}$ with a well-resolved three peak vibrational structure, while the second peak is centred at $368 \mathrm{~nm}$ and has two resolved vibrational peaks. This is true in solution as well, however the peak features are less apparent. ${ }^{10}$

Our simulations taking into account the vibrational effects in protein are in good agreement with the experimental spectra. Indeed, the vibrational structure and the peak broadening are well reproduced for both the first and second peaks. The QM/MM spectrum leads to a slight blue-shift of the first transition of around $10 \mathrm{~nm}$, and an important blue-shift of the second transition of $30-40 \mathrm{~nm}$. The spectra calculated in the protein are distinct from the spectra calculated in the gas phase, indicating that the electrostatic interaction and the structure confinement due to the protein scaffold are essential to capture the vibrational structure of the absorption spectrum. 

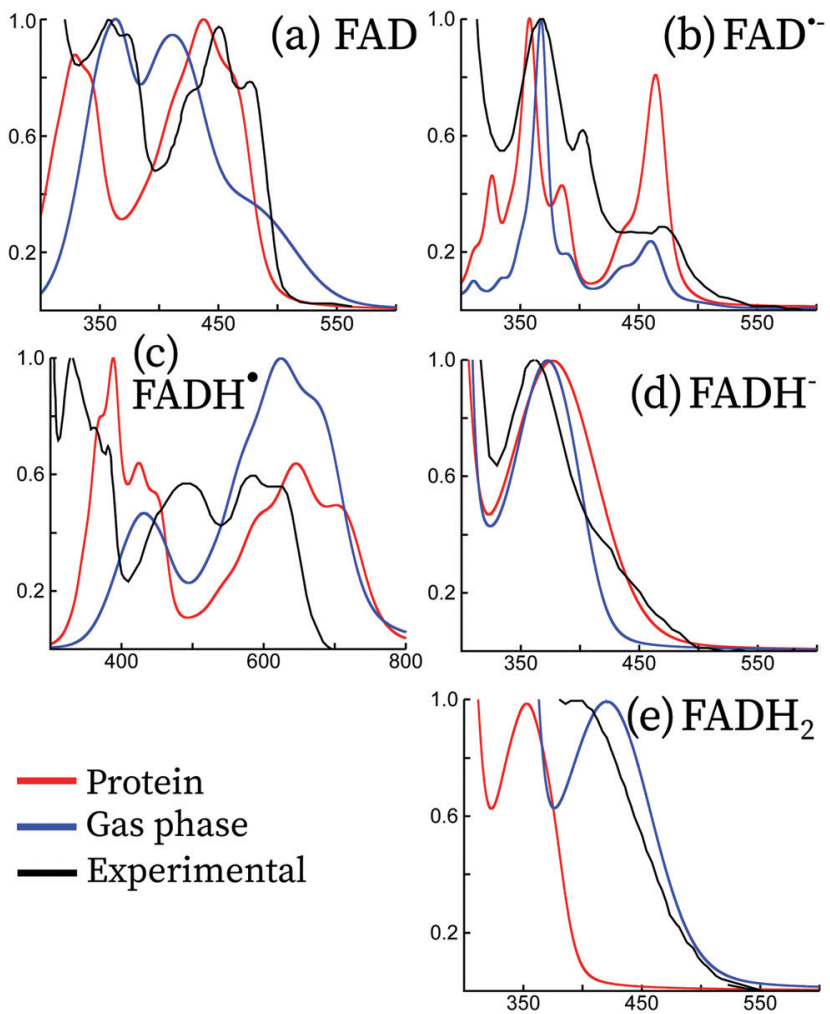

Fig. 5 Comparison between the experimental (black) and theoretical absorption spectra of the five redox forms of the flavin adenine dinucleotide. Vibrationally resolved theoretical spectra have been computed in the gas phase (blue) and in protein (red). For the sake of comparison, all spectra have been normalized to the most intense peak between $300 \mathrm{~nm}$ and $800 \mathrm{~nm}$. Experimental spectral data for FAD and FAD ${ }^{-}$have been taken from mosquito cryptochrome 1 (Anopheles gambiae), and FADH ${ }^{\bullet}$ and $\mathrm{FADH}^{-}$from a photolyase of $E$. Coli. The four forms are similar to the absorption spectrum of Arabidopsis Thaliana cryptochrome 1 and have been taken from ref. 45. The spectrum of $\mathrm{FADH}_{2}$ has been taken from solution measurements, and it is reproduced from the data taken from ref. 10

\section{FAD $^{\bullet-}$}

From Arabidopsis Thaliana cryptochrome 1 (AtCry1), an anion radical is obtained after $450 \mathrm{~nm}$ photoexcitation of FAD via an electron transfer from a tryptophan residue found nearby the protein cavity. ${ }^{65}$ For some animal cryptochromes it can even be the primary photoproduct after blue light irradiation. ${ }^{43,45}$ Its spectroscopic signatures are three clearly distinguished peaks at 372,402 and $490 \mathrm{~nm}^{40}$

From our results, we observe overall good agreement between the experimental and theoretical spectra. The transition energies in all the spectra range up to $300 \mathrm{~nm}$. In contrast, the intensities are only approximately reproduced, despite the vibrational structure being in good accordance with experimental spectra. On the one hand, the simulated absorption spectrum in protein largely overestimates the intensity of the lowest-energy peak, although the vibrational progression and broadening match well the experimental peak. The relative intensity obtained using the simulations in the gas phase better matches with that obtained using the experiment. On the other hand, the vibrational structure of the peaks above $400 \mathrm{~nm}$ is well reproduced by the simulations in protein, while the gas phase simulations do not correctly describe this region.

In the literature, it has been argued that the peak around $370 \mathrm{~nm}$ corresponding to $\pi_{1} \rightarrow \pi_{3}$ could arise or have a contribution from a tyrosil radical (TyrO $),{ }^{46,66}$ which would be formed by a deprotonation of tyrosil and an electron transfer to the tryptophane radical previously formed. ${ }^{42,66}$ In Fig. 6, we show the vibrationally resolved theoretical spectrum of tyrosine 429 in its radical form. The spectrum is shifted by about $30 \mathrm{~nm}$ with respect to the TyrO in solution. Indeed, the peak position matches the region of the $\pi_{1} \rightarrow \pi_{3}$ and it has a similar shape. However, our simulations of the $\mathrm{FAD}^{\bullet-}$ spectrum show a peak in the same region originating from an isoalloxazine transition. Therefore, our simulations are not able to confirm or refute the hypothesis that TyrO ${ }^{\bullet}$ is present in the absorption spectrum of FAD'.

\section{FADH $^{\bullet}$}

Absorption difference spectra clearly show the formation of the neutral radical in AtCry1. ${ }^{41,42,44}$ It can be obtained after proton transfer coming from $\mathrm{TrpH}^{\bullet+}$ to the anionic radical. Alternatively, the nearby aspartate Asp396 may also function as a proton donor. ${ }^{8}$ Müller et al. reported an absorption spectrum for AtCry1 extracted from a mixture of the oxidized FAD and neutral radical. ${ }^{46}$ Contrary to the oxidized and anionic radical forms, the neutral radical has a nonzero absorbance in the green/red visible spectral region, between 500 and $650 \mathrm{~nm}$.

Our simulations only qualitatively reproduce the absorption spectrum of $\mathrm{FADH}^{\circ}$, but the overall spectrum shows a large red shift of about $100 \mathrm{~nm}$ both for the gas phase and the protein embedded simulations with respect to experiments. We attribute this to the chosen level of theory, attributed to the lack of derivative discontinuity of the exchange-correlation potential,

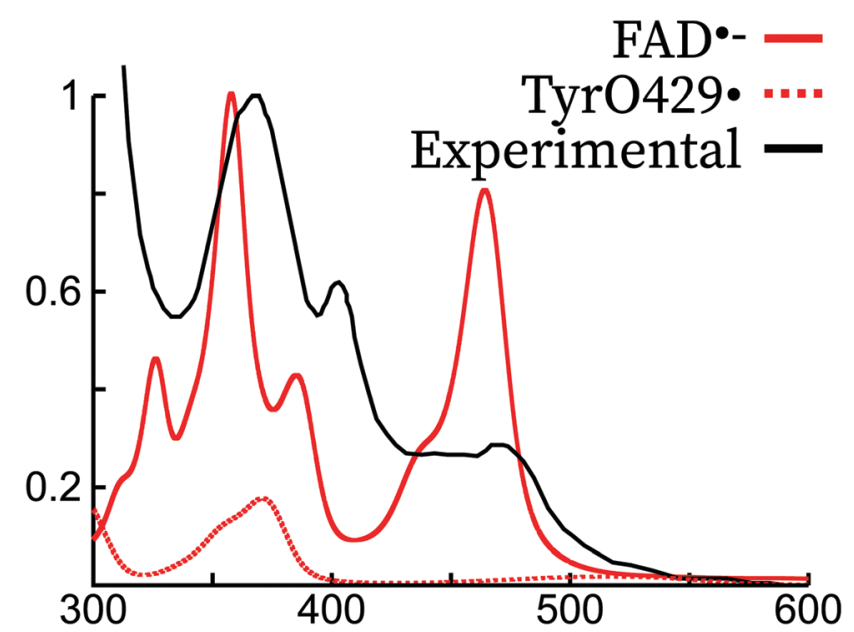

Fig. 6 Comparison of the experimental spectrum of FAD ${ }^{\bullet-}$ (ref. 45) with the theoretical spectrum of FAD ${ }^{--}$and Tyr429 ${ }^{\bullet}$ in the Arabidopsis Thaliana cryptochrome. The experimental and theoretical FAD ${ }^{\bullet-}$ spectra have been normalized to 1 for the largest intensity peak. The TyrO429 intensity was scaled with the same scaling factor as $F A D^{\bullet-}$, supposing thus a $1: 1$ mixture of the two species. 
which underestimates the fundamental gaps especially of radical systems. ${ }^{67}$ As far as the peak intensities are concerned, the protein simulations are in better accordance than the gas phase with respect to experiments. The simulations in protein seem to reproduce the relative intensities and the peak structure of the lowest energy and the highest energy peaks.

Both the gas phase and protein simulations completely miss the peak around $500 \mathrm{~nm}$. A peak was obtained in this region in previous theoretical simulations of $\mathrm{FADH}^{\bullet}$ in polar solvents using continuum models, ${ }^{7,13}$ but the origin is probably different from the peak appearing in protein. Rather, we attribute this peak as a radical signature of a tryptophan radical. In the experimental literature, it has been argued that a contribution from the Trp ${ }^{\bullet}$ spectrum is present in the absorption spectrum of $\mathrm{FADH}^{\bullet}{ }^{46,66}$ In Fig. 7, we show the shifted absorption spectrum of $\mathrm{FADH}^{\bullet}$ together with the spectrum of the deprotonated radical tryptophan409. As it can be observed, the position of the tryptophan spectrum falls in the region of $500 \mathrm{~nm}$, which would explain the extra peak missing in our simulated spectrum. The two peaks that were appearing in the $\mathrm{FADH}^{\bullet}$ spectrum would therefore correspond to the first and third peak in the experimental spectrum. Owing to the relative intensities of the $\mathrm{FADH}^{\bullet}$ and $\mathrm{Trp}^{\bullet}$, the concentration of both species would be $1: 1$, therefore excluding the formation of TyrO ${ }^{\bullet}$, which if formed from $\operatorname{Trp}^{\bullet}$ and would otherwise decrease its concentration in protein.

\section{FADH $^{-}$}

The fully reduced state is not favored in vivo and in vitro requires a strong reducing agent. ${ }^{68,69}$ In protein the reduced

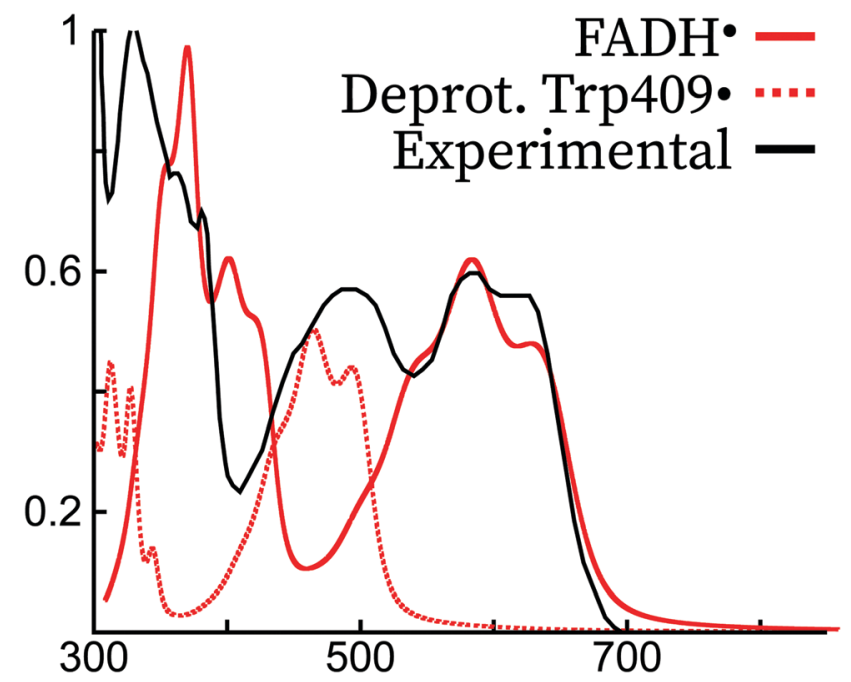

Fig. 7 Comparison of the experimental spectrum of $\mathrm{FADH}^{\bullet}(E$. Coli photolyase, ref. 45) with the theoretical spectrum of $\mathrm{FADH}^{\bullet}$ and $\operatorname{Trp409}{ }^{\bullet}$ in the Arabidopsis Thaliana cryptochrome. The experimental and theoretical $\mathrm{FADH}^{\bullet}$ spectra have been normalized to 1 for the largest intensity peak, and the theoretical spectrum of $\mathrm{FADH}^{\bullet}$ was blue-shifted by ca. $70 \mathrm{~nm}$ to match the most intense peak of the lowest energy experimental transition. Trp409 ${ }^{\circ}$ was not shifted, and its intensity was scaled with the same scaling factor as $\mathrm{FADH}^{\bullet}$, supposing thus a $1: 1$ mixture of the two species. anion is formed after a second electron transfer from tryptophan, usually after a second photon absorption from $\mathrm{FADH}^{\bullet} .^{70}$ Reoxidation occurs in the presence of molecular oxygen. In solution, the anionic reduced form has a maximum absorption peak at $256 \mathrm{~nm}$ and shoulders at 288 and $350 \mathrm{~nm} .{ }^{71}$ A study in the $E$. Coli photolyase showed an absorption band around $360 \mathrm{~nm}$, with very weak absorbance in the visible region. ${ }^{10,45}$

Our simulations both in protein and in the gas phase match the experimental spectrum, both in terms of shape and vibrational progression. In the experimental spectrum, a shoulder appears at around $450 \mathrm{~nm}$, which is probably due to some residual species (like a small concentration of $\operatorname{Trp}^{\bullet}$ ) or an almost dark $\pi \rightarrow \pi^{*}$ transition as found in the experimental Stark spectra and theoretical simulations of $\mathrm{FADH}^{-}$in solution. $^{13,72}$ This feature is not captured by our QM/MM simulations, probably due to an underestimation of the intensity of this transition.

\section{FADH $_{2}$}

The neutral reduced form is formed after a second proton transfer from tryptophan from the $\mathrm{FADH}^{-}$form. In protein, its spectrum hardly differs from $\mathrm{FADH}^{-43}$ and its characterization is mostly limited to solution studies, where it has a distinguished shoulder at $400 \mathrm{~nm} .^{10,71}$

The experimental measurements for this form shown in Fig. 5 were done in solution. These match well with the gasphase calculations, but the protein calculations show a blueshift of this peak of around $100 \mathrm{~nm}$, leading to a similar excitation energy to that of $\mathrm{FADH}^{-}$. This large blue-shift does not have an electrostatic origin, but it can be rather attributed to the enforced planar structure due to the steric hindrance of the protein, since the electronic transition of $\mathrm{FADH}_{2}$ in the gas phase at the protein optimized conformation leads to the same absorption maximum. Unfortunately, there is no clear experimental evidence of this form in the cryptochrome protein to determine whether this shift of the QM/MM simulations is realistic.

\section{Conclusions}

The simulations of the UV-visible absorption spectrum in complex media are a difficult task in theoretical chemistry. Here, we have developed and implemented the analytic secondderivatives of the electrostatic embedding QM/MM ESPF method $^{24}$ for unrestricted wavefunctions, extending our previous developments ${ }^{17,23}$ to open-shell systems. This method allows, among other molecular properties, the vibrational analysis of minimum energy structures and the simulations of the vibrationally resolved spectra in protein. We have applied this methodology to simulate the absorption spectrum of FAD and its four redox derivatives (two radicals and two reduced forms, one of each anionic and one neutral) embedded in the Arabidopsis Thaliana cryptochrome protein.

The theoretical absorption spectrum in protein shows a perfect agreement with the experimental spectrum for the 
non-radical forms, reproducing well both the intensity, peak position and vibrational peak structure of the electronic transitions found between $300 \mathrm{~nm}$ and $600 \mathrm{~nm}$. In contrast, the agreement in the case of the radical forms is only moderate. As discussed recently in the literature, ${ }^{46,66}$ the signature of tryptophan and tyrosine radicals could be found in the absorption spectra of $\mathrm{FAD}^{\bullet-}$ and $\mathrm{FADH}^{\bullet}$. On the one hand, for $\mathrm{FAD}^{\bullet-}$, a tyrosil peak appears at the same position corresponding to the $\pi \rightarrow \pi^{*}$ transition. The position and shape of both peaks are similar, and therefore we cannot conclude that a tyrosil signature is present. On the other hand, the theoretical $\mathrm{FADH}^{\bullet}$ absorption spectrum in protein can only be matched to the experimental spectrum considering the lowest intense peak of the $\operatorname{Trp}^{\bullet}$ radical at around $500 \mathrm{~nm}$. We estimate the proportion between the two species as $1: 1$.

In conclusion, we demonstrate that vibrational analysis at the electrostatic embedding QM/MM level is a valuable tool to interpret the complex absorption spectra arising in proteins. In the future, we plan to extend our method to the simulations of vibrational spectroscopy (infra-red, non-resonant Raman, etc.) as well as extending the vibrational analysis to other kinds of wavefunctions.

\section{Conflicts of interest}

There are no conflicts to declare.

\section{Acknowledgements}

The authors acknowledge financial support from the "Agence Nationale pour la Recherche" through the project BIOMAGNET (ANR-16CE29-0008-01). "Centre de Calcul Intensif d'AixMarseille" is acknowledged for granting access to its high performance computing resources.

\section{Notes and references}

1 X. Yu, H. Liu, J. Klejnot and C. Lin, The Arabidopsis Book, 2010, vol. 2010.

2 S. Braatsch and G. Klug, Photosynth. Res., 2004, 79, 45-57.

3 I. Chaves, R. Pokorny, M. Byrdin, N. Hoang, T. Ritz, K. Brettel, L.-O. Essen, G. T. J. van der Horst, A. Batschauer and M. Ahmad, Annu. Rev. Plant Biol., 2011, 62, 335-364.

4 C. Lin, D. Top, C. C. Manahan, M. W. Young and B. R. Crane, Proc. Natl. Acad. Sci. U. S. A., 2018, 115, 3822-3827.

5 M. Liedvogel and H. Mouritsen, J. R. Soc., Interface, 2010, 7, S147-S162.

6 A. Pinzon-Rodriguez, S. Bensch and R. Muheim, J. R. Soc., Interface, 2018, 15, 20180058.

7 P. Mondal, K. Schwinn and M. Huix-Rotllant, J. Photochem. Photobiol., A, 2020, 387, 112164.

8 I. A. Solov'yov, T. Domratcheva, A. R. Moughal Shahi and K. Schulten, J. Am. Chem. Soc., 2012, 134, 18046-18052.

9 P. Mondal and M. Huix-Rotllant, Phys. Chem. Chem. Phys., 2019, 21, 8874-8882.
10 Y.-T. Kao, C. Saxena, T.-F. He, L. Guo, L. Wang, A. Sancar and D. Zhong, J. Am. Chem. Soc., 2008, 130, 13132-13139.

11 B. Klaumünzer, D. Kröner and P. Saalfrank, J. Phys. Chem. B, 2010, 114, 10826-10834.

12 Y. Ai, C. Zhao, J. Xing, Y. Liu, Z. Wang, J. Jin, S. Xia, G. Cui and X. Wang, J. Phys. Chem. A, 2018, 122, 7954-7961.

13 M. P. Kabir, Y. Orozco-Gonzalez and S. Gozem, Phys. Chem. Chem. Phys., 2019, 21, 16526-16537.

14 F. Müller, Free Radical Biol. Med., 1987, 3, 215-230.

15 E. Cannuccia, O. Pulci, R. D. Sole and M. Cascella, Chem. Phys., 2011, 389, 35-38.

16 C. Nielsen, M. S. Norby, J. Kongsted and I. A. Solov'yov, J. Phys. Chem. Lett., 2018, 9, 3618-3623.

17 K. Schwinn, N. Ferré and M. Huix-Rotllant, J. Chem. Theory Comput., 2020, DOI: 10.1021/acs.jctc.9b01145.

18 S. Dapprich, I. Komáromi, K. Byun, K. Morokuma and M. J. Frisch, J. Mol. Struct. THEOCHEM, 1999, 461-462, 1-21. 19 Q. Cui and M. Karplus, J. Chem. Phys., 2000, 112, 1133-1149. 20 Q. Cui and M. Karplus, J. Phys. Chem. B, 2000, 104, 3721-3743.

21 A. Ghysels, H. L. Woodcock, J. D. Larkin, B. T. Miller, Y. Shao, J. Kong, D. V. Neck, V. V. Speybroeck, M. Waroquier and B. R. Brooks, J. Chem. Theory Comput., 2011, 7, 496-514.

22 T. Giovannini, L. Grazioli, M. Ambrosetti and C. Cappelli, J. Chem. Theory Comput., 2019, 15, 5495-5507.

23 K. Schwinn, N. Ferré and M. Huix-Rotllant, J. Chem. Phys., 2019, 151, 041102.

24 N. Ferré and J. G. Ángyán, Chem. Phys. Lett., 2002, 356, 331-339. 25 F. Melaccio, M. Olivucci, R. Lindh and N. Ferré, Int. J. Quantum Chem., 2011, 111, 3339-3346.

26 J. A. Pople, R. Krishnan, H. B. Schlegel and J. S. Binkley, Int. J. Quantum Chem., 1979, 16, 225-241.

27 M. J. Frisch, G. W. Trucks, H. B. Schlegel, G. E. Scuseria, M. A. Robb, J. R. Cheeseman, G. Scalmani, V. Barone, G. A. Petersson, H. Nakatsuji, X. Li, M. Caricato, A. V. Marenich, J. Bloino, B. G. Janesko, R. Gomperts, B. Mennucci, H. P. Hratchian, J. V. Ortiz, A. F. Izmaylov, J. L. Sonnenberg, D. Williams-Young, F. Ding, F. Lipparini, F. Egidi, J. Goings, B. Peng, A. Petrone, T. Henderson, D. Ranasinghe, V. G. Zakrzewski, J. Gao, N. Rega, G. Zheng, W. Liang, M. Hada, M. Ehara, K. Toyota, R. Fukuda, J. Hasegawa, M. Ishida, T. Nakajima, Y. Honda, O. Kitao, H. Nakai, T. Vreven, K. Throssell, J. A. Montgomery, Jr., J. E. Peralta, F. Ogliaro, M. J. Bearpark, J. J. Heyd, E. N. Brothers, K. N. Kudin, V. N. Staroverov, T. A. Keith, R. Kobayashi, J. Normand, K. Raghavachari, A. P. Rendell, J. C. Burant, S. S. Iyengar, J. Tomasi, M. Cossi, J. M. Millam, M. Klene, C. Adamo, R. Cammi, J. W. Ochterski, R. L. Martin, K. Morokuma, O. Farkas, J. B. Foresman and D. J. Fox, Gaussian 16 Revision A.01, Gaussian Inc., Wallingford CT, 2016.

28 J. W. Ponder, TINKER: Software tools for molecular design, version 6.3, 2010, http://dasher.wustl.edu/tinker/.

29 A. D. Becke, J. Chem. Phys., 1993, 98, 5648-5652.

30 C. Lee, W. Yang and R. G. Parr, Phys. Rev. B: Condens. Matter Mater. Phys., 1988, 37, 785-789. 
31 S. H. Vosko, L. Wilk and M. Nusair, Can. J. Phys., 1980, 58, 1200-1211.

32 A. Schäfer, H. Horn and R. Ahlrichs, J. Chem. Phys., 1992, 97, 2571-2577.

33 A. Schäfer, C. Huber and R. Ahlrichs, J. Chem. Phys., 1994, 100, 5829-5835.

34 K. Eichkorn, O. Treutler, H. Öhm, M. Häser and R. Ahlrichs, Chem. Phys. Lett., 1995, 240, 283-290.

35 K. Eichkorn, F. Weigend, O. Treutler and R. Ahlrichs, Theor. Chem. Acc., 1997, 97, 119-124.

36 D. A. Case, I. Y. Ben-Shalom, S. R. Brozell, D. S. Cerutti, T. E. Cheatham, III, V. W. D. Cruzeiro, T. A. Darden, R. E. Duke, D. Ghoreishi, M. K. Gilson, H. Gohlke, A. W. Goetz, D. Greene, R. Harris, N. Homeyer, S. Izadi, A. Kovalenko, T. Kurtzman, T. S. Lee, S. LeGrand, P. Li, C. Lin, J. Liu, T. Luchko, R. Luo, D. J. Mermelstein, K. M. Merz, Y. Miao, G. Monard, C. Nguyen, H. Nguyen, I. Omelyan, A. Onufriev, F. Pan, R. Qi, D. R. Roe, A. Roitberg, C. Sagui, S. Schott-Verdugo, J. Shen, C. L. Simmerling, J. Smith, R. Salomon-Ferrer, J. Swails, R. C. Walker, J. Wang, H. Wei, R. M. Wolf, X. Wu, L. Xiao, D. M. York and P. A. Kollman, AMBER 2018, University of California, San Francisco, 2018.

37 J. Antony, D. Medvedev and A. Stuchebrukhov, J. Am. Chem. Soc., 2000, 122, 1057.

38 A. Baiardi, J. Bloino and V. Barone, J. Chem. Theory Comput., 2013, 9, 4097-4115.

39 P. Macak, Y. Luo and H. Ågren, Chem. Phys. Lett., 2000, 330, 447-456.

40 Y. S. Choong and V. Massey, J. Biol. Chem., 1980, 255, 8672-8677.

41 C. Lin, D. Robertson, M. Ahmad, A. Raibekas, M. Jorns, P. Dutton and A. Cashmore, Science, 1995, 269, 968-970.

42 B. Giovani, M. Byrdin, M. Ahmad and K. Brettel, Nat. Struct. Mol. Biol., 2003, 10, 489-490.

43 S.-H. Song, B. Dick, A. Penzkofer, R. Pokorny, A. Batschauer and L.-O. Essen, J. Photochem. Photobiol., B, 2006, 85, 1-16.

44 T. Kottke, A. Batschauer, M. Ahmad and J. Heberle, Biochemistry, 2006, 45, 2472-2479.

45 B. Liu, H. Liu, D. Zhong and C. Lin, Curr. Opin. Plant Biol., 2010, 13, 578-586.

46 P. Müller, J.-P. Bouly, K. Hitomi, V. Balland, E. D. Getzoff, T. Ritz and K. Brettel, Sci. Rep., 2014, 4, 5175.

47 P. Macheroux, UV-Visible Spectroscopy as a Tool to Study Flavoproteins, Humana Press, 1999, pp. 1-7.

48 J. Koziol, Experientia, 1965, 21, 189-190.

49 J. Koziol, Photochem. Photobiol., 1966, 5, 41-54.
50 J. Koziol, Photochem. Photobiol., 1969, 9, 45-53.

51 A. Weigel, A. L. Dobryakov, M. Veiga and J. L. P. Lustres, J. Phys. Chem. A, 2008, 112, 12054-12065.

52 P. Zirak, A. Penzkofer, T. Mathes and P. Hegemann, Chem. Phys., 2009, 358, 111-122.

53 T. Kottke, J. Heberle, D. Hehn, B. Dick and P. Hegemann, Biophys. J., 2003, 84, 1192-1201.

54 E. Sikorska, I. V. Khmelinskii, J. Koput, J. L. Bourdelande and M. Sikorski, J. Mol. Struct., 2004, 697, 137-141.

55 A. Penzkofer, J. Photochem. Photobiol., A, 2016, 314, 114-124.

56 C. B. Martin, X. Shi, M.-L. Tsao, D. Karweik, J. Brooke, C. M. Hadad and M. S. Platz, J. Phys. Chem. B, 2002, 106, 10263-10271.

57 M. Takahashi, Y. Ishikawa, J.-I. Nishizawa and H. Ito, Chem. Phys. Lett., 2005, 401, 475-482.

58 S. Salzmann, J. Tatchen and C. M. Marian, J. Photochem. Photobiol., A, 2008, 198, 221-231.

59 S. Salzmann and C. M. Marian, Chem. Phys. Lett., 2008, 463, 400-404.

60 J.-Y. Hasegawa, S. Bureekaew and H. Nakatsuji, J. Photochem. Photobiol., A, 2007, 189, 205-210.

61 M. M. N. Wolf, C. Schumann, R. Gross, T. Domratcheva and R. Diller, J. Phys. Chem. B, 2008, 112, 13424-13432.

62 C. Neiss, P. Saalfrank, M. Parac and S. Grimme, J. Phys. Chem. A, 2003, 107, 140-147.

63 M. Dittric, P. L. Freddolino and K. Schulten, J. Phys. Chem. $B, 2005,109,13006-13013$.

64 Y.-K. Choe, S. Nagase and K. Nishimoto, J. Comput. Chem., 2007, 28, 727-739.

65 K. Maeda, A. J. Robinson, K. B. Henbest, H. J. Hogben, T. Biskup, M. Ahmad, E. Schleicher, S. Weber, C. R. Timmel and P. J. Hore, Proc. Natl. Acad. Sci. U. S. A., 2012, 109, 4774-4779.

66 F. Lacombat, A. Espagne, N. Dozova, P. Plaza, P. Müller, K. Brettel, S. Franz-Badur and L.-O. Essen, J. Am. Chem. Soc., 2019, 141, 13394-13409.

67 E. J. Baerends, O. V. Gritsenko and R. van Meer, Phys. Chem. Chem. Phys., 2013, 15, 16408-16425.

68 P. Müller and M. Ahmad, J. Biol. Chem., 2011, 286, 21033-21040.

69 L. J. van Wilderen, G. Silkstone, M. Mason, J. J. van Thor and M. T. Wilson, FEBS Open Bio., 2015, 5, 885-892.

70 J. Wang, X. Du, W. Pan, X. Wang and W. Wu, J. Photochem. Photobiol., C, 2015, 22, 84-102.

71 P. Hemmerich, C. Veeger and H. C. S. Wood, Angew. Chem., 1965, 4, 671-687.

72 R. F. Pauszek, G. Kodali, M. S. U. Siddiqui and R. J. Stanley, J. Am. Chem. Soc., 2016, 138, 14880-14889. 